\title{
A review of blood transfusions in a trauma unit for young children
}

\author{
M Salverda, ${ }^{1} \mathrm{MB}$ ChB; N Ketharanathan, ${ }^{1} \mathrm{MD} ; \mathrm{M}$ van Dijk,${ }^{1,2} \mathrm{PhD} ;$ E Beltchev, ${ }^{3} \mathrm{MB}$ ChB; H Buys, ${ }^{3} \mathrm{FCP} ;$ A Numanoglu, ${ }^{2}$ FSC; \\ A B van $\mathbf{A s},{ }^{2} \mathrm{PhD}$ \\ ${ }^{1}$ Department of Intensive Care and Pediatric Surgery, Erasmus MC Sophia Children's Hospital, Rotterdam, Netherlands \\ ${ }^{2}$ Department of Paediatric Surgery, Faculty of Health Sciences, University of Cape Town and Red Cross War Memorial Children's Hospital, \\ Cape Town, South Africa \\ ${ }^{3}$ Department of Paediatrics and Child Health, Faculty of Health Sciences, University of Cape Town and Red Cross War Memorial Children's \\ Hospital, Cape Town, South Africa
}

Corresponding author: A B van As (sebastian.vanas@uct.ac.za)

\begin{abstract}
Background. Trauma is the leading cause of mortality and morbidity worldwide. Blood transfusions play an incremental role in the acute phase, yet practice varies owing to variations in transfusion thresholds and concerns about potential complications, especially in children. Objectives. To evaluate protocol adherence to blood transfusion thresholds in paediatric trauma patients and determine the degree of blood product wastage, as defined by discarded units.

Methods. A retrospective, descriptive study of trauma patients (age 0 - 13 years) who received a blood transfusion in the trauma unit at Red Cross War Memorial Children's Hospital, Cape Town, South Africa, over a 5.5-year period (1 January 2009 - 1 July 2014). Haemoglobin $(\mathrm{Hb})$ transfusion thresholds were defined as $10 \mathrm{~g} / \mathrm{dL}$ for neurotrauma patients and patients requiring skin grafting or a musculocutaneous flap (group 1). All other trauma patients had an Hb transfusion threshold of $7 \mathrm{~g} / \mathrm{dL}$ (group 2).

Results. A total of 144 patients were included (mean age 5.2 years (standard deviation (SD) 3.3), 68.1\% male). The mean Hb increase after transfusion was $3.5 \mathrm{~g} / \mathrm{dL}$ (SD 1.7). Adherence to the transfusion $\mathrm{Hb}$ threshold protocol was $96.7 \%$ for group 1 v. $34.0 \%$ for group 2 . No complications were reported. Average blood wastage was 3.5 units per year during the study period.

Conclusions. Adherence to paediatric blood transfusion protocol was low in the Hb threshold group $<7 \mathrm{~g} / \mathrm{dL}$. However, transfusion-related complications and wastage were minimal. Further prospective research is required to determine optimal blood transfusion guidelines for paediatric trauma patients.
\end{abstract}

S Afr Med J 2017;107(3):227-231. DOI:10.7196/SAMJ.2017.v107i3.11307

Trauma is the leading cause of death in the paediatric population. ${ }^{[1]}$ Red blood cell (RBC) transfusions can play a lifesaving role in these patients, but doctors are often reluctant to administer blood transfusions to children because of potential complications. In a prospective study involving adult trauma patients, Malone et al. ${ }^{[2]}$ demonstrated that receiving a blood transfusion is one of the predictors of mortality. Other predictors of mortality in trauma patients include intensive care unit (ICU) admission, ICU length of stay and hospital length of stay in trauma patients. The association of blood transfusions with adverse outcome in trauma patients is probably because injured children requiring a blood transfusion tend to be more critically ill than those who do not need one.

Non-infectious adverse outcomes associated with blood transfusion include allergic reactions, immunomodulation, volume overload, transfusion-related acute lung injury and haemolysis. ${ }^{[3]}$ It is therefore important to ensure patient safety by developing, implementing and regulating blood transfusion protocols. Research performed over the past decade has shown that the pre-transfusion haemoglobin $(\mathrm{Hb}) /$ haematocrit $(\mathrm{Hct})$ threshold could be related to adverse outcomes. The optimal $\mathrm{Hb}$ threshold for trauma patients is still unknown. ${ }^{[4,5]}$ A study of paediatric RBC transfusion practice in the $\mathrm{UK}^{[6]}$ demonstrated that the median pre-transfusion $\mathrm{Hb}$ level was $7.9 \mathrm{~g} / \mathrm{dL}$, varying by diagnosis. Lacroix et al. ${ }^{[7]}$ documented that an $\mathrm{Hb}$ threshold of $7 \mathrm{~g} / \mathrm{dL}$ for RBC transfusions reduced transfusion requirements without increasing adverse outcomes. Further- more, a recent study involving orthopaedic trauma patients (aged 18 - 50 years $)^{[8]}$ suggested that liberal transfusion practices could pose an additional risk. This report showed that receiving a transfusion increased the risk of immune-related adverse events $(p<0.01)$.

The high number of trauma cases in South Africa (SA) and the consequent high requirement for blood transfusions have resulted in recurrent shortages of blood products. We therefore studied current transfusion practices in one of the busiest paediatric trauma centres in SA, Red Cross War Memorial Children's Hospital (RCWMCH) in Cape Town, a public children's hospital.

\section{Objectives}

The first objective of this study was to evaluate whether RCWMCH uses blood transfusions appropriately in paediatric trauma patients. Appropriate use was defined by the current RCWMCH transfusion guideline that only children with an $\mathrm{Hb}$ value $<7 \mathrm{~g} / \mathrm{dL}$ should receive a blood transfusion. Exception is made for all children who have suffered a head injury, or require a skin graft or musculocutaneous flap procedure. The occurrence of secondary brain injury in paediatric neurotrauma patients can be reduced by securing adequate cerebral circulation and oxygenation, in which blood transfusion plays a crucial role. It is also assumed that blood supply affects the success rate in skin grafts and musculocutaneous flap procedures. In the RCWMCH trauma unit, an $\mathrm{Hb}$ threshold of $10 \mathrm{~g} / \mathrm{dL}$ is therefore used in these cases. 
The second objective was to analyse the difference between the number of RBC units ordered and the number actually transfused in the trauma unit, to study potential wastage of blood products.

The third objective was to record complications and mortality in young children who received a blood transfusion.

\section{Methods}

A retrospective chart review was performed of all trauma patients who received an RBC transfusion in the trauma unit at RCWMCH between 1 January 2009 and 1 July 2014. Ethical approval was obtained from the Human Research Ethics Committee of the University of Cape Town (ref. no. 549/2014). The RCWMCH blood bank database was used to identify patients for whom RBCs were ordered.

RCWMCH is a public secondary- and tertiary-level dedicated children's hospital in sub-Saharan Africa. It admits approximately 10000 trauma patients aged $<13$ years annually. The patients were selected for this study according to the following criteria: an Abbreviated Injury Score (AIS) of 3 or 4 (Table 1), and transfusion of RBCs in the trauma ward within 1 week after the incident. Patient characteristics extracted from the RCWMCH registry included age, gender, mechanism of injury, diagnosis, $\mathrm{Hb}$ and Hct levels, volume of RBCs ordered, volume of RBCs administered, blood pressure, pulse, Glasgow Coma Score (GCS), capillary refill time, complications, treatment and AIS.

The patients' weight was estimated on admission as their clinical condition did not permit formal measurement. The Advanced Paediatric Life Support (APLS) formula was applied: Estimated weight $(\mathrm{kg})=($ Age +4$) \times 2$. An SA study involving emergency patients at RCWMCH showed that the APLS method is one of the most accurate in estimating weight in the Western Cape paediatric population, even though it has a slight tendency to underestimate weight. ${ }^{[7]}$

Cape Town is home to a very diverse population, and several different languages (the main ones being isiXhosa, Afrikaans and English) are spoken in the drainage area of the hospital. To assess which language group received the most transfusions, we also documented the language.

\begin{tabular}{|c|c|}
\hline 1 (mild) & $\begin{array}{l}\text { Patients with minor injuries, } \\
\text { patients discharged home }\end{array}$ \\
\hline \multirow[t]{5}{*}{2 (moderate) } & $\begin{array}{l}\text { Patients with wounds requiring } \\
\text { suturing or any other procedure }\end{array}$ \\
\hline & Patients requiring theatre \\
\hline & Patients with fractures \\
\hline & Patients admitted \\
\hline & $\begin{array}{l}\text { Patients with minor and } \\
\text { intermediate head injuries }\end{array}$ \\
\hline \multirow[t]{5}{*}{3 (severe) } & Polytraumatised patients \\
\hline & Shocked patients \\
\hline & Patients requiring resuscitation \\
\hline & $\begin{array}{l}\text { Patients with severe head } \\
\text { injuries (deeply comatose and/or } \\
\text { intubated) }\end{array}$ \\
\hline & Patients requiring intensive care \\
\hline 4 (death) & Patients who died \\
\hline
\end{tabular}

The RCWMCH blood bank database was used to compute quantities of blood ordered and transfused. The difference between these two numbers represented wastage of blood products in that specific case. The term 'waste of blood products' was defined as the discarding of least one full unit of blood.

Although numerous blood products can be transfused (including platelets, factor 8, etc.), in this study we focused on the more common types, since the other products are rarely administered in the trauma setting. There are three of these, each with its own specific product code: (i) whole blood; (ii) adult RBCs; and (iii) paediatric RBCs (PRBCs). Whole blood is increasingly rarely used and its clinical indications are limited. The few patients who did receive whole blood were therefore excluded from the study. RBCs referred to adult units, each of which contained a mean of $300 \mathrm{~mL}$ (standard deviation (SD) 50) of RBCs. One unit of PRBCs contained a mean of $130 \mathrm{~mL}$ (SD 25) of RBCs. The net cost of one RBC unit ranged from ZAR582.46 in 2009 to ZAR890.35 in 2014. If un-crossmatched RBCs were cancelled before their delivery they were not considered wasted, since these units could still be used for other patients.

\section{Subgroups}

We assigned the patients to one of two subgroups based on the pretransfusion $\mathrm{Hb}$ threshold. Group 1, the ' $\mathrm{Hb}<10 \mathrm{~g} / \mathrm{dL}$ group', included all patients with a head injury or who had undergone a surgical procedure in which an adjusted $\mathrm{Hb}$ threshold was crucial for the reasons given above. Group 2, the ' $\mathrm{Hb}<7 \mathrm{~g} / \mathrm{dL}$ group', included all other patients.

\section{Statistical analysis}

The retrospective data were entered into an SPSS database and analysed using SPSS version 22 (IBM, USA). Normally distributed variables were described as means with SDs, and non-normally distributed variables were presented as medians with interquartile ranges (IQRs).

\section{Results}

The blood bank transfusion database contained 728 patients, of whom 285 were identified as having received one or more RBC transfusions over the 5.5-year study period. Of these patients, 22 had an AIS of $\leq 2$. Another 110 patients were excluded because their medical record folder was incomplete or unavailable. A further 9 were excluded because they were transfused $>1$ week after the primary traumatic

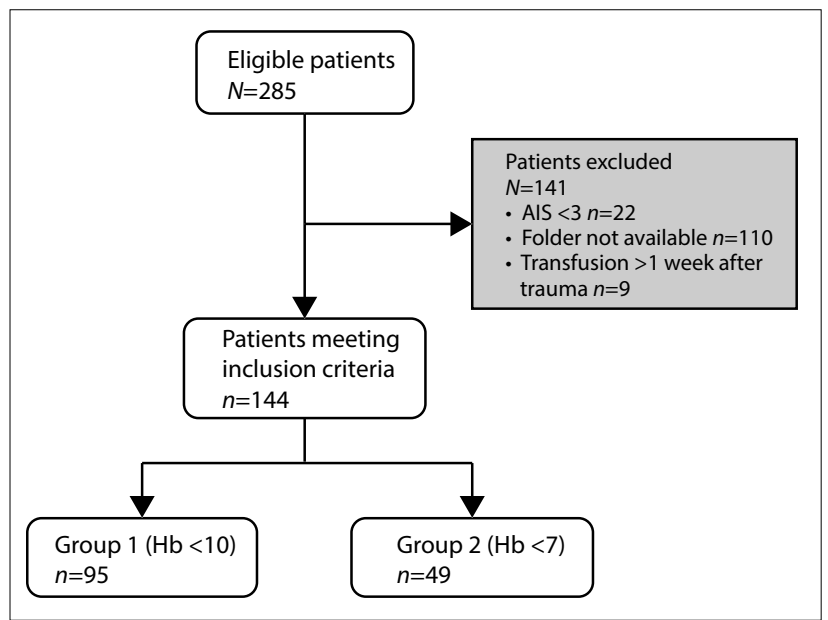

Fig. 1. Flowchart of patients excluded from the study and those who met the inclusion criteria. 
injury. After excluding these 141 patients, 144 children (19.7\%) met the inclusion criteria (Fig. 1).

Of these children, 95 were included in group 1, of whom 91 had their pre-transfusion $\mathrm{Hb}$ recorded, and 49 were in group 2, of whom 47 patients had their pre-transfusion $\mathrm{Hb}$ recorded.

The patient characteristics are set out in Table 2. The mean age of the children was 5.2 years (SD 3.3). The majority (68.1\%) were male. Both the pre-transfusion and post-transfusion $\mathrm{Hb}$ values were available for $93.1 \%$ of the patients. The mean post-transfusion $\mathrm{Hb}$ value ( $n=137$ patients) was $11.0 \mathrm{~g} / \mathrm{dL}$ (SD 1.5), corresponding with a mean increase in $\mathrm{Hb}$ after transfusion of $3.5 \mathrm{~g} / \mathrm{dL}$ (SD 1.7).

\section{Subgroup analysis}

Transfusion practice by $\mathrm{Hb}$ transfusion threshold

The mean pre-transfusion $\mathrm{Hb}$ value in group $1(\mathrm{Hb}$ transfusion threshold $<10 \mathrm{~g} / \mathrm{dL}$ ) and group 2 (threshold $<7 \mathrm{~g} / \mathrm{dL}$ ) was $7.5 \mathrm{~g} /$ $\mathrm{dL}$ (SD 1.6 and SD 1.5, respectively). Group 1 had a mean posttransfusion $\mathrm{Hb}$ value of $11.2 \mathrm{~g} / \mathrm{dL}$ (SD 1.5), and group 2 a mean of $10.7 \mathrm{~g} / \mathrm{dL}$ (SD 1.4).

The scatterplots in Fig. 2 (A and B) demonstrate the $\mathrm{Hb}$ values over time in groups 1 and 2, respectively. The horizontal dashed lines in the figure represent the $\mathrm{Hb}$ threshold for transfusion as per local protocol. In group 1, 3 of the 91 patients (3.3\%) received an $\mathrm{RBC}$ transfusion, although their $\mathrm{Hb}$ values before transfusion were $>10 \mathrm{~g} / \mathrm{dL}$. In group 2, 31 of the 47 transfused patients (66.0\%) had a pre-transfusion $\mathrm{Hb}$ value above the threshold of $7.0 \mathrm{~g} / \mathrm{dL}$. Of these 31 children, 26 required surgery while the other 5 had a degree of critical ischaemia around the injury or wound. It appeared that clinical decisions made by the trauma staff determined the ordering and transfusion of blood products in these instances.

\section{Transfusion volume by body weight}

The estimated weights of the study patients ranged from $8.3 \mathrm{~kg}$ to $33.9 \mathrm{~kg}$, with a mean of $18.4 \mathrm{~kg}$ (SD 6.5). The transfusion volume for RBCs in the paediatric population is usually $10-15 \mathrm{~mL} / \mathrm{kg}$ body weight and not age dependent. The median transfused blood volume for the total group $(N=144)$ was $14.5 \mathrm{~mL} / \mathrm{kg}$ (IQR 9.9 - 19.7). Fig. 3 demonstrates the transfused blood volume based on estimated body weight for groups 1 and 2 . In general, the median transfusion volume for both groups was $\sim 15 \mathrm{~mL} / \mathrm{kg}$ (group 1: $14.3 \mathrm{~mL} / \mathrm{kg}$ (IQR 9.9 - 20.5) and group 2: $15.2 \mathrm{~mL} / \mathrm{kg}$ (IQR 9.4 - 18.6)).

\section{Blood product wastage}

During the 5.5-year study period, 11 potential cases of blood product wastage were identified, with a total of 19 units of RBCs. Eight of these 11 patients were admitted to the trauma ward when the RBC units were ordered. The number of wasted units per patient ranged

\section{Table 2. Patient characteristics}

\begin{tabular}{ll}
\hline Variables & All patients $(\mathbf{N = 1 4 4 )}$ \\
\hline Sex, $n$ (\%) & $98(68.1)$ \\
Male & $46(31.9)$ \\
Female & \\
Age (yr) & $4.5(2.6-7.1)$ \\
Median (IQR) & $0.2-12.9$ \\
Range & \\
First language, $n(\%)$ & $47(33.6)$ \\
English & $37(26.4)$ \\
Afrikaans & $55(39.3)$ \\
Xhosa & $1(0.7)$ \\
Sesotho & \\
Admission vital signs, $n(\%)$ & $142(98.6)$ \\
CFT normal & $2(1.4)$ \\
CFT $>3$ s & $38(27.0)$ \\
GCS $\leq 8$ & \\
Mechanism of injury, $n(\%)$ & $77(53.5)$ \\
MVA pedestrian & $22(15.3)$ \\
MVA passenger & $4(2.8)$ \\
Burns & $7(4.9)$ \\
Fall & $14(9.8)$ \\
Physical assault & $8(5.6)$ \\
Gunshot & $2(1.4)$ \\
Dog bite & $10(6.9)$ \\
Other & \\
CFT capillary refill time; MVA = motor vehicle accident. \\
\end{tabular}

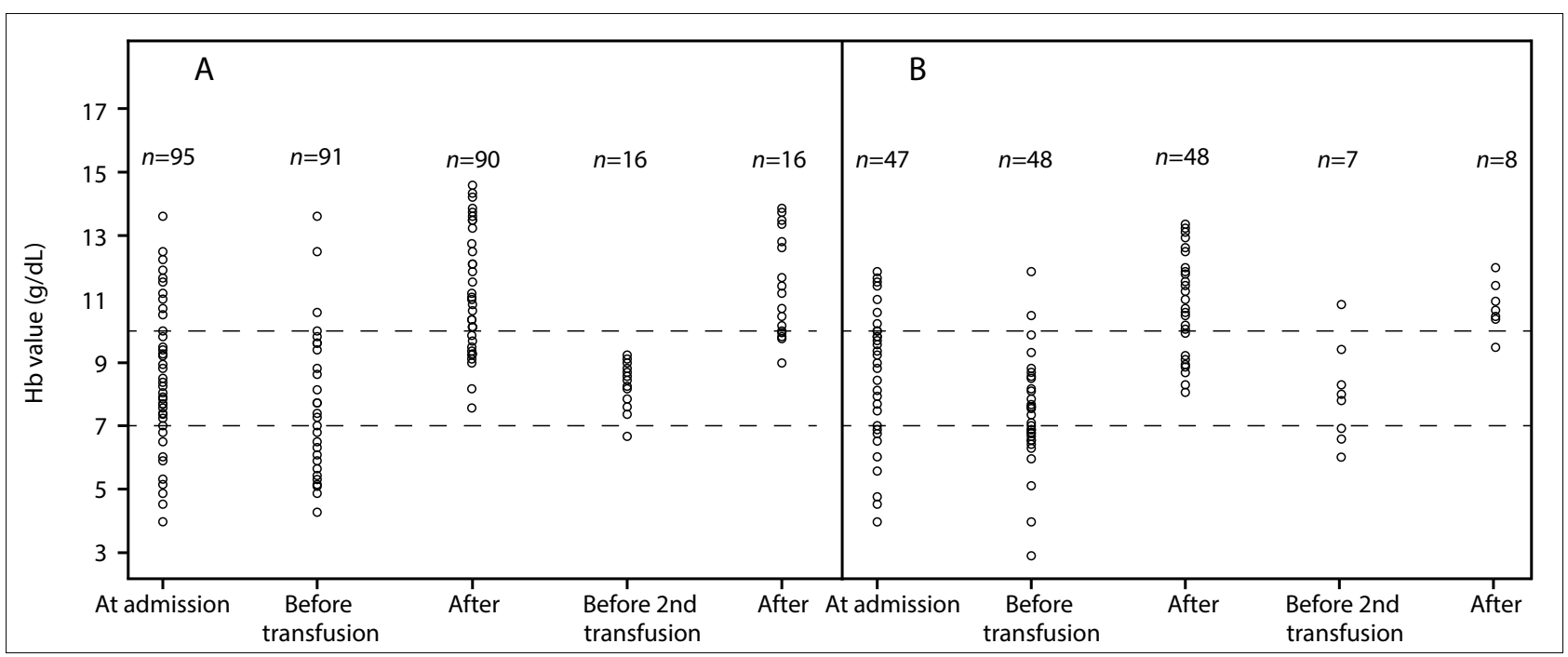

Fig. 2. Mean $\mathrm{Hb}$ values before and after transfusion in groups 1 (A) and 2 (B). (Horizontal dashed lines $=H b$ threshold for transfusion as per local protocol.) 


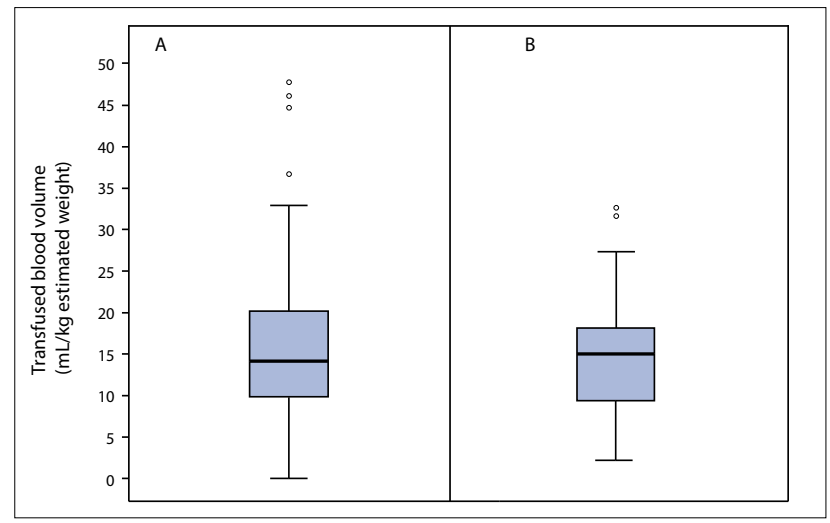

Fig. 3. Median volume of blood transfused for groups $1(A)$ and $2(B)$.

from 1 to 6 . In the case in which 6 units were wasted, an 11-year-old boy had sustained rupture of the liver and vena cava inferior in a motor vehicle accident (MVA). A total of 9 RBC units were ordered, but according to the medical records he received 3 units. Four units were ordered preoperatively with the note 'If needed'. According to the medical folder, these units were neither given nor cancelled, so they were considered wasted. According to the blood bank database, another 2 units were ordered but there was no record in the patient's hospital folder of these units having been given. These units were therefore also considered wasted. In general, average blood wastage was 3.5 units per year during the study period.

\section{Complications and mortality}

No transfusion-related complications were reported. During the study period, 2 patients $(1.4 \%)$ died, both as a result of lethal traumatic head injury. The first non-survivor was a 1-year-old comatose boy $($ GCS $<8)$ who had been involved in an MVA. He received a blood transfusion in theatre on the day of admission. The pre-transfusion $\mathrm{Hb}$ value was $5.0 \mathrm{~g} / \mathrm{dL}$ and the post-transfusion value $14.2 \mathrm{~g} / \mathrm{dL}$. He died in the ICU 3 days after admission. The second non-survivor was a 7-year-old boy who had also been involved in an MVA, sustaining multiple injuries, and died on the day of admission. His pre-transfusion $\mathrm{Hb}$ value was $5.4 \mathrm{~g} / \mathrm{dL}$, and his post-transfusion value was not recorded.

\section{Discussion}

Blood transfusions play a lifesaving role in the stabilisation of patients with severe trauma. This study evaluated adherence to RBC transfusion protocols in paediatric trauma patients at RCWMCH in sub-Saharan Africa, a region with a high incidence of paediatric trauma. Our results demonstrated that adherence to local protocol regarding the $\mathrm{Hb}$ transfusion threshold of $7 \mathrm{~g} / \mathrm{dL}$ (group 2) is low, with the majority of these patients $(66.0 \%)$ receiving an $\mathrm{RBC}$ transfusion despite their $\mathrm{Hb}$ being $>7 \mathrm{~g} / \mathrm{dL}$. The volume of blood transfused was within the estimated need range for most cases. No complications were reported, and blood product wastage (defined as discarded units) was minimal.

\section{Transfusion policy adherence}

It is of interest to explore the difference in transfusion protocol adherence between group $1(\mathrm{Hb}<10 \mathrm{~g} / \mathrm{dL})$ and group $2(\mathrm{Hb}<7 \mathrm{~g} /$ $\mathrm{dL}$ ). It can be speculated that a lower transfusion threshold is more easily overruled by clinical decision-making to create an ' $\mathrm{Hb}$ margin' in a trauma patient when ongoing blood loss is suspected and/ or surgery is necessary. Furthermore, the protocol leaves it to the trauma team on call to assess requirement for blood transfusions in patients with head injuries and critical wounds. This deviation from policy is in agreement with several reports in the literature ${ }^{[7,9,10]}$ and in line with Vincent $e t$ al., ${ }^{[11]}$ who reported that adult patients admitted for emergency surgery were commonly transfused (57.5\%). Despite patients above the transfusion threshold frequently receiving transfusions, the volume of blood transfused was within the estimated need range $(10-20 \mathrm{~mL} / \mathrm{kg})$ in most cases.

\section{Blood wastage}

Given the limited number of blood donors, the expense of blood products and the short half-life of RBCs, every effort should be made to prevent unnecessary transfusions. Nineteen wasted blood units in 11 patients were identified between 2009 and 2014, giving an average amount of 3.5 discarded blood units per year and indicating prudent use. Taking into consideration the severity of injuries suffered by these 11 patients, including extensive traumatic brain injury, and the indication for acute emergency surgical intervention, this wastage could be considered acceptable and may indicate a conscientious practice of ordering and transfusion of blood products. However, it can also be argued that a large number of patients received a blood transfusion that was not technically indicated given that they had an $\mathrm{Hb}$ value above the transfusion threshold. Although the blood product was used and not discarded, this could be regarded as a form of blood product wastage.

\section{Study limitations}

This study was retrospective in design, and data were collected from paper medical records. This inevitably resulted in missing data, limiting the number of patients who could be evaluated. Furthermore, information on discarded RBC units was not specifically noted in the patient records. If there was no documentation in the patient folder that the ordered RBC units were actually given, these units were considered wasted. This could have led to overestimation of blood product wastage. Another potential limitation is the necessity to estimate patient weight. Although studies have shown a good correlation between calculated weight and actual weight with the APLS formula, it cannot be ruled out that this could have over- or underestimated blood volume transfusion calculations.

Overall, our observations indicate that transfusion practice is often clinically guided and that information about clinical decision-making is difficult (if not impossible) to collect retrospectively, leading to speculation about protocol deviations.

\section{Conclusions}

This study provided insight into blood transfusion practice in paediatric trauma patients at RCWMCH. The RCWMCH approach was not based on strict adherence to the blood transfusion protocol, especially in the case of the $\mathrm{Hb}$ transfusion threshold of $7 \mathrm{~g} / \mathrm{dL}$. Instead, the number of RBCs ordered and transfused appeared to be determined mainly by each individual case. Blood product wastage and complications were negligible. The results of this retrospective study encourage us to perform future prospective, randomised trials to investigate the optimal $\mathrm{Hb}$ threshold and possible predictors of higher transfusion requirements in the acute treatment of paediatric trauma patients.

\footnotetext{
1. Nosanov L, Inaba K, Okoye O, et al. The impact of blood product ratios in massively transfused pediatric trauma patients. Am J Surg 2013;206(5):655-660. http://dx.doi.org/10.1016/j.amjsurg. 2013.07.009

2. Malone DL, Dunne J, Tracy JK, Putnam AT, Scalea TM, Napolitano LM. Blood transfusion, independent of shock severity, is associated with worse outcome in trauma. J Trauma 2003;54(5):898905; discussion 905-897. http://dx.doi.org/10.1097/01.TA.0000060261.10597.5C

Istaphanous GK, Wheeler DS, Lisco SJ, Shander A. Red blood cell transfusion in critically ill children: A narrative review. Pediatr Crit Care Med 2011;12(2):174-183. http://dx.doi.org/10.1097/ PCC.0b013e3181e30d09
} 
4. Boutin A, Chasse M, Shemilt M, et al. Red blood cell transfusion in patients with traumatic brain injury: A systematic review and meta-analysis. Transfus Med Rev 2016;30(1):15-24. http://dx.doi. org/10.1016/j.tmrv.2015.08.004

Goel R, Cushing MM, Tobian AA. Pediatric patient blood management programs: Not just transfusing little adults. Transfus Med Rev 2016;30(4):235-241. http://dx.doi.org/10.1016/j.tmrv.2016.07.004

6. New HV, Grant-Casey J, Lowe D, Kelleher A, Hennem S, Stanworth SJ. Red blood cell transfusion practice in children: Current status and areas for improvement? A study of the use of red blood cell transfusions in children and infants. Transfusion 2014;54(1):119-127. http://dx.doi.org/10.1111/ trf. 12313

7. Lacroix J, Hebert PC, Hutchison JS, et al. Transfusion strategies for patients in pediatric intensive care units. N Engl J Med 2007;356(16):1609-1619. http://dx.doi.org/10.1056/NEJMoa066240

8. Mullis B, Fisk E, Weaver D, Zhao Q, Daggy I, di Cesare PE. Anemia versus transfusion: Does blood conservation increase the risk of complications? Am J Orthop (Belle Mead NJ) 2015;44(1):E11-E16.
9. Fortin S, Cardona LG, Latreille M, Tucci M, Lacroix J. Blood transfusion in acute and chronic pediatric settings: Beliefs and practices. Transfusion 2016;56(1):130-138. http://dx.doi.org/10.1111/trf.13352 10. Willems A, Harrington K, Lacroix J, et al. Comparison of two red-cell transfusion strategies after pediatric cardiac surgery: A subgroup analysis. Crit Care Med 2010;38(2):649-656. http://dx.doi. pediatric cardiac surgery: A subgroup

org/10.1097/CCM.0b013e3181bc816c
11. Vincent JL, Baron JF, Reinhart K, et al. Anemia and blood transfusion in critically ill patients. JAMA 2002;288(12):1499-1507. http://doi.org/10.1001/jama.288.12.1499 